Recepción: 15 / 04 / 2017

Ciencias Administrativas

Aceptación: 01 / 05 / 2017

Articulo Científico

Publicación: 15 / 05 / 2017

\title{
La gestión administrativa de las empresas de transporte urbano de Babahoyo y sus efectos en el grado de satisfacción de los usuarios
}

\author{
The administrative management of the urban transport companies of Babahoyo \\ and its effects on the degree of satisfaction of the users
}

\section{A gestão administrativa das empresas de transporte urbano Babahoyo e seu efeito sobre o grau de satisfação do usuario}

\author{
Wendy L. Ocampo-Ulloa \\ wocampo@utb.edu.ec
}
Washington Pazmiño-Gavilanez" wpazmino@utb.edu.ec

\section{Veronica A. Merchan-Jacome vmerchan@utb.edu.ec}

Correspondencia: wocampo@utb.edu.ec

\footnotetext{
Magister en Administración de Empresas, Universidad Técnica Estatal de Quevedo UTEQ; Ingeniera en Empresas y Administración de Negocios; Tecnóloga Administradora de Empresas; Técnica Ejecutiva en Negocios; Universidad Regional Autónoma de los Andes UNIANDES, Docente de la Universidad Técnica de Babahoyo. Ecuador.

II. Magister En Dirección Estratégica, Universidad Regional Autónoma de los Andes; Ingeniero Comercial, Universidad Técnica de Babahoyo. Docente de la Universidad Técnica de Babahoyo. Ecuador

III. Magister en Administración de Empresas, Universidad Técnica de Babahoyo; Economista, Universidad de Guayaquil. Docente de la Universidad Técnica de Babahoyo. Ecuador
} 


\section{Resumen}

El transporte terrestre en el Ecuador es considerado como un sector estratégico, esto se debe a que diariamente miles de personas deben hacer uso de este medio de transporte para poder movilizarse hacia distintos lugares. Las Cooperativas de Trasporte Urbano SANTA RITA y FLUMINENSE presta el servicio de transportar a los ciudadanos dentro del perímetro urbano de la ciudad de Babahoyo diariamente a distintos lugares.

Bajo este contexto se propone la necesidad de analizar la gestión administrativa y sus efectos en la satisfacción de los usuarios, periodo 2011-2012; que permita recabar información pertinente para detectar las falencias dentro de la administración y proponer los cambios necesarios para así poder competir dentro del mercado de la transportación.

El presente estudio permitió identificar las crecientes necesidades de los ciudadanos que utilizan el transporte público como medio de movilización, las cuales serán analizadas y mediante estrategias implementadas en la Gestión Administrativa aprovechando al máximo los recursos en especial el talento humano ya que son ellos quienes a diariamente tratan con el usuario al momento de transportarlo de un sitio a otro.

Palabras clave: Gestión administrativa; transporte urbano; grado de satisfacción. 
Wendy L. Ocampo-Ulloa; Washington Pazmiño-Gavilanez; Veronica A. Merchan-Jacome

\begin{abstract}
Land transportation in Ecuador is considered as a strategic sector, this is due to the fact that thousands of people must make use of this means of transport daily to be able to move to different places. The Urban Transport Cooperatives SANTA RITA and FLUMINENSE provide the service of transporting the citizens within the urban perimeter of the city of Babahoyo daily to different places.
\end{abstract}

In this context, it is proposed to analyze the administrative management and its effects on user satisfaction, period 2011-2012; That allows to gather pertinent information to detect the failures within the administration and to propose the necessary changes in order to be able to compete within the market of the transport.

The present study made it possible to identify the growing needs of citizens who use public transport as a means of mobilization, which will be analyzed and through strategies implemented in Administrative Management taking full advantage of resources, especially human talent, since they are the ones who daily Deal with the user when transporting it from one site to another.

Key words: Administrative management; urban transport; Degree of satisfaction. 


\section{Resumo}

Transporte terrestre no Equador é considerado como um setor estratégico, este é porque cada milhares dia das pessoas deve fazer uso deste meio de transporte para se deslocar para lugares diferentes. As cooperativas de transporte urbano e FLUMINENSE SANTA RITA fornece o serviço de transporte de cidadãos dentro dos limites da cidade da cidade de Babahoyo diariamente em lugares diferentes.

Neste contexto, a necessidade de analisar a gestão administrativa e seu impacto na satisfação do usuário, período 2011-2012 é proposta; reunir informações pertinentes que permitam detectar as falhas na administração e propor mudanças necessárias para competir no mercado para o transporte.

Este estudo identificou as necessidades crescentes dos cidadãos que utilizam os transportes públicos como meio de mobilização, que serão analisados e através de estratégias implementadas na administração fazendo o melhor uso dos recursos talento especial humanos e eles são os únicos a diária lidar com o usuário quando se deslocam de um lugar para outro.

Palavras chave: Gerenciamento administrativo; transporte urbano; grau de satisfação. 
Wendy L. Ocampo-Ulloa; Washington Pazmiño-Gavilanez; Veronica A. Merchan-Jacome

\section{Introducción.}

Esta investigación se justifica en razón de que, los objetivos que se buscan alcanzar con una buena gestión administrativa de las Empresas de Transporte Urbano, es que los servicios sean operados de manera confiable, eficiente y al menor costo posible, para esto es importante una adecuada reglamentación de las funciones que deban regir los procesos administrativos. Si no existe una adecuada gestión, se manejaran incorrectamente los servicios, lo que en consecuencia afectará negativamente la satisfacción del usuario de transporte urbano. (Heredia Bedoya, 2015)

En el competitivo mundo de la transportación de pasajeros, es necesario responder a las exigencias de los usuarios, con un análisis adecuado de los riesgos internos y externos que están afectando a las empresas de transporte urbano para buscar consolidar la imagen de las mismas mediante la presentación de un nuevo concepto en el servicio de la transportación, aplicando una correcta gestión administrativa que garantice la operatividad razonable. (Ortúzar \& Willumsen, 2008) (Egaña Ugrinovic, Ortúzar, \& Rizzi, 2009)

Una buena gestión administrativa empieza con los recursos humanos, esto encierra aspectos de capacitación, pues ellos son los encargados de ejecutar el servicio, y dar el mantenimiento de las unidades que también es un aspecto importante para garantizar el cumplimiento de las normas nacionales y la calidad que esperan los clientes. Si no se gestiona adecuadamente al personal se perderá calidad en el servicio de transporte urbano. (Yáñez, Raveau, \& Ortúzar, 2010)

La calidad del servicio está dada por la frecuencia con la que transitan, el respeto del prestador del servicio hacia el usuario y el estado de la unidad, si se cumplen estos tres aspectos el usuario estará satisfecho y por ende el propietario mantiene su rentabilidad. De no cumplirse estos aspectos necesarios el usuario no estará satisfecho con el servicio. (Ortúzar \& Román, 2003) 
La gestión administrativa de las empresas de transporte urbano de Babahoyo y sus efectos en el grado de satisfacción de los usuarios

Para lograr un buen servicio a la ciudadanía es necesario que la empresa tenga un buen manejo de sus recursos y sus socios se sientan complacidos con los beneficios que le otorga estar agrupados en una cooperativa, así se sentirán estimulados a mejorar el servicio para el usuario.

Por lo expuesto, es pertinente realizar un estudio de la gestión administrativa que aplica la cooperativa para adecuado manejo de sus recursos humano, económico y material que influya positivamente en la opinión que el usuario tiene del servicio de transporte urbano.

\section{Materiales y métodos.}

En la presente investigación se utilizó varios métodos, técnicas, instrumentos, procedimientos y actividades que sirvieron como medio para alcanzar los objetivos propuestos.

Deductivo.- este método permitió puntualizar cómo se maneja los procedimientos administrativos en las empresas de transporte urbano, y las causas por las que se da cumplimiento a la planificación.

Descriptivo.- ayudo a determinar los aspectos más relevantes sobre el funcionamiento de las empresas de transporte urbano de la ciudad de Babahoyo, sus fortalezas y debilidades.

Cualitativo.- permitió determinar y estudiar las diferentes características y cualidades que tienen las empresas, estableciendo la forma de trabajo de los empleados que están encargados del manejo de la información.

\section{Técnicas de la investigación}

Se buscó obtener información directa mediante las siguientes herramientas: 
Wendy L. Ocampo-Ulloa; Washington Pazmiño-Gavilanez; Veronica A. Merchan-Jacome

Observación directa.- mediante esta técnica, el investigador asistió a las diferentes áreas de las empresas de transporte urbano de la ciudad de Babahoyo para así verificar los procesos administrativos.

Entrevista.- con esta técnica se procedió a realizar un banco de preguntas que tiene relación con el planteamiento de la hipótesis, para lo cual se entrevistó a los gerentes quienes son los encargados de la administración de las empresas de transporte urbano.

Encuesta.- esta técnica sirvió para examinar toda la información, se aplicaron encuestas a los empleados y usuarios de las empresas de transporte urbano de la ciudad de Babahoyo.

\section{Construcción metodológica del objeto de investigación}

El tema que fue objeto de investigación, fue elegido por ser una problemática actual, latente por mucho tiempo y que merece especial atención pues es parte del diario vivir del ciudadano. Por este motivo se efectuaron encuestas, ya que es el mejor procedimiento para obtener los datos requeridos para la investigación. En esta incluí datos generales así como preguntas cerradas para mayor especificación en la obtención de resultados.

La investigación se hizo mediante un cuestionario que fue utilizado como parte del estudio. El mismo constó de aseveraciones y preguntas que fueron contestadas individualmente por cada uno de los miembros de la muestra seleccionada.

Siendo nuestra población total de 22500 personas, incluyendo al personal administrativo como a los usuarios a quienes las cooperativas de transporte urbano prestan su servicio, aplicando la formula estadística se obtuvo la muestra a investigar que fue de 377 personas. 
La gestión administrativa de las empresas de transporte urbano de Babahoyo y sus efectos en el grado de satisfacción de los usuarios

Obtenida esta información se procedió a realizar la tabulación, diagnóstico y análisis de los resultados obtenidos, el mismo que permitió conocer toda la información necesaria para poder desarrollar este trabajo de investigación.

\section{Determinación de los instrumentos}

Son los recursos que permitieron la obtención de la información requerida para llevar a cabo la investigación.

Los instrumentos utilizados fueron:

El trabajo de campo para el desarrollo de este estudio fue realizado en los meses de agosto a noviembre del 2011.

Se incluyeron las siguientes actividades:

Se solicitó autorización a las cooperativas de transporte urbano de la ciudad de Babahoyo para poder realizar la investigación

Se suministró el cuestionario al personal administrativo y a los usuarios en general, para el desarrollo del estudio.

Se procedió al análisis y tabulación de los datos, luego realizada la interpretación de los datos, se llegaron a unas conclusiones.

De acuerdo a los resultados obtenidos, se analizó la necesidad de plantear una propuesta alternativa de trabajo para la organización. El mismo incluye posibles recomendaciones que serán de utilidad para la empresa. 
Wendy L. Ocampo-Ulloa; Washington Pazmiño-Gavilanez; Veronica A. Merchan-Jacome

Se entrevistó a los gerentes de las dos cooperativas de transporte urbano existentes en la ciudad de Babahoyo, para conocer cuáles son criterios referentes al tema de investigación.

Se realizó un cuestionario de preguntas para los usuarios en general a quienes se les presta el servicio de transportación para así medir su satisfacción acerca del servicio.

El cuestionario es un instrumento básico de la observación en la encuesta y en la entrevista, en él se desarrollaron una serie de preguntas las cuales permitieron medir una o más variables en esta investigación.

\section{Población}

La población de estudio fue de 22500 usuarios que son los que reciben el servicio que prestan las cooperativas de transporte urbano; 377.71 (Muestra de usuarios)

\section{Resultados.}

\section{1.- ¿Qué tipo de transporte utiliza para movilizarse con más frecuencia?}

\section{Cuadro 1.- TIPO DE TRANSPORTE UTILIZADO CON FRECUENCIA}

\begin{tabular}{|l|c|c|}
\hline OPCIONES & FRECUENCIA & $\%$ \\
\hline Taxi & 49 & $13 \%$ \\
\hline Selectivo & 294 & $78 \%$ \\
\hline Automóvil & 34 & $9 \%$ \\
\hline Otro & 0 & 0 \\
\hline Total & 377 & $100 \%$ \\
\hline
\end{tabular}

Un $78 \%$ de las personas expresó que viaja en selectivo con mayor frecuencia que en otros tipos de transporte. Mientras que un 13\% y 9\% viajan en taxi y automóvil respectivamente. Quiere decir que las personas son principalmente usuarios del transporte urbano, que de cualquier otro tipo de transporte. 


\section{2.- Durante el día ¿Con que frecuencia, utiliza este medio de transporte?}

\section{Cuadro 2.- FRECUENCIA CON QUE UTILIZAN UN MEDIO DE TRANSPORTE}

\begin{tabular}{|l|c|c|}
\hline OPCIONES & FRECUENCIA & $\%$ \\
\hline $\mathbf{1}$ vez & 0 & 0 \\
\hline $\mathbf{2}$ veces & 60 & $16 \%$ \\
\hline 3 veces & 144 & $38 \%$ \\
\hline $\mathbf{4}$ veces & 75 & $26 \%$ \\
\hline $\mathbf{5}$ veces & 98 & $20 \%$ \\
\hline 6 veces & 0 & 0 \\
\hline Total & 377 & 100 \\
\hline
\end{tabular}

Un $38 \%$ de las personas respondieron que tres veces usan un medio de transporte, y un $26 \%$, $20 \%$ y $16 \% 5$ veces, 4 veces, 2 veces respectivamente. La frecuencia con que las personas usan algún tipo de transporte, torna a este servicio en algo necesario para la vida de los usuarios.

3.- De los medios de transporte que utiliza, cual considera usted, es el más contaminante y que puede afectar su salud:

\section{Cuadro 3.- MEDIO DE TRANSPORTE CONSIDERADO COMO MÁS CONTAMINANTE}

\begin{tabular}{|l|c|c|}
\hline OPCIONES & FRECUENCIA & $\%$ \\
\hline Taxi & 0 & 0 \\
\hline Selectivo & 339 & $90 \%$ \\
\hline Automóvil & 38 & $10 \%$ \\
\hline Otro & 0 & 0 \\
\hline Total & 377 & $100 \%$ \\
\hline
\end{tabular}

Un 90\% manifestó que considera al selectivo como el medio de transporte más contaminante que puede afectar su salud, solo un $10 \%$ dijo que considera al automóvil como el medio de transporte más contaminante. Queda claro que los usuarios han apreciado que los selectivos son los medios más contaminantes y peligrosos para el medio ambiente y su salud. 
4.- ¿En cuál de los transportes que utiliza usted, corre más riesgos de ser objeto de violencia, robo, o maltrato?

\section{Cuadro 4.- MEDIO DE TRANSPORTE EN EL QUE SE CORRE MÁS RIESGOS}

\begin{tabular}{|l|c|c|}
\hline OPCIONES & FRECUENCIA & $\%$ \\
\hline Taxi & 11 & $3 \%$ \\
\hline Selectivo & 287 & $76 \%$ \\
\hline Automóvil & 79 & $21 \%$ \\
\hline Otro & 0 & 0 \\
\hline Total & 377 & 100 \\
\hline
\end{tabular}

Un $76 \%$ de los usuarios dice que el medio de transporte donde corren más riesgos de ser objeto de violencia, robo, o maltrato, es el selectivo. Por otro lado un $21 \%$ y $3 \%$ expresó que el automóvil y el taxi respectivamente. Esto indica que los usuarios son mayormente objetos de violencia, robo o maltrato en un selectivo de transportación urbana que en cualquier otro medio de transporte.

5.- ¿Cómo califica usted la calidad de servicio que prestan las cooperativas de transporte urbano a los usuarios?

Cuadro 5.- CALIDAD DEL SERVICIO DE COOPERATIVAS TRANSPORTE URBANO

\begin{tabular}{|l|c|c|}
\hline OPCIONES & FRECUENCIA & $\%$ \\
\hline Muy Bueno & 15 & $4 \%$ \\
\hline Regular & 260 & $69 \%$ \\
\hline Bueno & 102 & $27 \%$ \\
\hline Total & 377 & 100 \\
\hline
\end{tabular}

El 69\% de las personas consideran regular la calidad del servicio que prestan las cooperativas de transporte urbano, mientras que un $27 \%$ y un $4 \%$ consideran como bueno y muy bueno respectivamente. Lo que apunta a que el servicio que prestan las cooperativas de transporte urbano, no es de la total satisfacción del usuario, y que debe mejorar. 
6.- ¿Tomando en cuenta el valor que se cobra por el servicio de transporte urbano, y la calidad del servicio que prestan, el costo es:

Cuadro 6.- RELACION ENTRE EL VALOR Y LA CALIDAD DEL SERVICIO DE TRANSPORTE URBANO

\begin{tabular}{|l|c|c|}
\hline OPCIONES & FRECUENCIA & $\%$ \\
\hline Económico & 57 & $15 \%$ \\
\hline Muy caro & 214 & $57 \%$ \\
\hline Barato & 106 & $28 \%$ \\
\hline Total & 377 & 100 \\
\hline
\end{tabular}

El $57 \%$ de los usuarios consideran como muy caro el valor del servicio en relación a la calidad del mismo, y un $28 \%$ y $15 \%$ lo consideran barato y económico respectivamente. Es notorio que las deficiencias en el servicio de transportación urbana, hacen que el usuario juzgue como que está pagando mucho dinero, teniendo en cuenta el mal servicio que recibe.

7.- El tiempo que demora el transporte urbano hasta llegar a su parada, lo considera usted:

Cuadro 7.- TIEMPO QUE DEMORA EL TRANSPORTE URBANO PARA LLEGARA UNA PARADA

\begin{tabular}{|l|c|l|}
\hline OPCIONES & FRECUENCIA & $\%$ \\
\hline Optimo & 64 & $17 \%$ \\
\hline Rápido & 166 & $44 \%$ \\
\hline Lento & 147 & $39 \%$ \\
\hline Total & 377 & 100 \\
\hline
\end{tabular}

Un $44 \%$ de las personas expresaron que es rápido el recorrido hasta su parada, y un 39\% y $17 \%$ piensan que es lento y optimo respectivamente. Se observa que existe una mínima diferencia entre los dos porcentajes superiores, una gran parte de los usuarios opinan que el recorrido de las unidades es rápido, y el otro opinan que es lento. Tal vez se deba a que las unidades algunas veces 
avanzan muy acelerado por retraso de turno o simple competencia por pasajeros y en otras lento por esperar más pasajeros.

8.- ¿Cómo califica usted las condiciones en las que se encuentran las unidades que prestan el servicio de transporte urbano?

Cuadro 8.- CONDICIONES DE LAS UNIDADES DE TRANSPORTE URBANO SEGÚN OPINION DE USUARIOS

\begin{tabular}{|l|c|c|}
\hline OPCIONES & FRECUENCIA & $\%$ \\
\hline Muy buenas & 11 & $3 \%$ \\
\hline Buenas & 204 & $54 \%$ \\
\hline Malas & 162 & $43 \%$ \\
\hline Total & 377 & 100 \\
\hline
\end{tabular}

Un 54\% de los usuarios calificaron como buenas las condiciones de las unidades de transporte urbano, y un $43 \%$ y $3 \%$ las califican de malas y muy buenas respectivamente. Se observa que existe una mínima diferencia, entre los dos primeros porcentajes, seguramente esto obedece a que algunas unidades si dan mantenimiento y limpieza a sus unidades, mientras que en otras no lo hacen.

9.- ¿Está usted de acuerdo con el trato que se le da a los grupos vulnerables dentro de un medio de transporte urbano?

Cuadro 9.-CONFORMIDAD CON EL TRATO DADO A LOS GRUPOS VULNERABLES

\begin{tabular}{|l|c|c|}
\hline OPCIONES & FRECUENCIA & $\%$ \\
\hline SI & 185 & $49 \%$ \\
\hline NO & 192 & $51 \%$ \\
\hline Total & 377 & 100 \\
\hline
\end{tabular}


Un 51\% de los usuarios no está de acuerdo con el trato que se le da a los grupos vulnerables dentro de las unidades de transporte, y un $49 \%$ si está de acuerdo. Es evidente que existe una opinión dispar con el asunto del trato a las personas de tercera edad, niños, mujeres embarazadas y discapacitados, pero el hecho es que existe malestar, porque la mayoría no está contenta con el trato que recibe el usuario.

Encuesta dirigida al personal administrativo de las Cooperativas de Transporte Urbano de la Ciudad de Babahoyo.

1.- ¿Conoce la misión de la cooperativa en que usted labora?

Cuadro 10.- CONOCIMIENTO DE LA MISIÓN DE LA COOPERATIVA DE TRANSPORTE URBANO

\begin{tabular}{|l|c|l|}
\hline OPCIONES & FRECUENCIA & $\%$ \\
\hline SI & 68 & $48 \%$ \\
\hline NO & 75 & $52 \%$ \\
\hline Total & 143 & 100 \\
\hline
\end{tabular}

Un 52\% del personal administrativo si conoce la misión de la cooperativa, y un $48 \%$ no la conoce. Esto indica la falta de conocimiento de gran parte del personal respecto a los fundamentos esenciales para una efectiva administración de una empresa de servicio, la misión es la razón de ser de toda organización.

2.- ¿Cree que está contribuyendo con el cumplimiento de la misma? 
Wendy L. Ocampo-Ulloa; Washington Pazmiño-Gavilanez; Veronica A. Merchan-Jacome

\section{Cuadro 11.- CUMPLIMIENTO DE LA MISION SEGÚN OPINION DEL PERSONAL}

\begin{tabular}{|l|c|c|}
\hline OPCIONES & FRECUENCIA & $\%$ \\
\hline SI & 68 & $48 \%$ \\
\hline NO & 75 & $52 \%$ \\
\hline Total & 143 & 100 \\
\hline
\end{tabular}

Un 52\% del personal administrativo si cree contribuir con el cumplimiento de la misión de la cooperativa, y un $48 \%$ cree no hacerlo. La falta de conocimiento de la misión que persigue la empresa, es motivo para no contribuir al cumplimiento de la misma.

3.- ¿Lo estimulan para que realice bien su trabajo (con capacitaciones, reconocimientos, etc.)?

Cuadro 12.- ESTIMULO POR EL TRABAJO REALIZADO

\begin{tabular}{|l|c|c|}
\hline OPCIONES & FRECUENCIA & $\%$ \\
\hline SI & 66 & $46 \%$ \\
\hline NO & 77 & $54 \%$ \\
\hline Total & 143 & 100 \\
\hline
\end{tabular}

Un 54\% del personal administrativo expresa que si se les estimula por realizar bien su trabajo, y un $46 \%$ piensa que no. La variación en ambas respuestas denota que no todos están siendo debidamente estimulados para realizar su trabajo de manera eficiente.

\section{4.- ¿Mantienen el gerente buenas relaciones con el personal, incluido usted?}

\section{Cuadro 13.- BUENAS RELACIONES ENTRE GERENTE Y PERSONAL}

\begin{tabular}{|l|c|c|}
\hline OPCIONES & FRECUENCIA & $\%$ \\
\hline SI & 143 & $100 \%$ \\
\hline NO & 0 & 0 \\
\hline Total & 143 & 100 \\
\hline
\end{tabular}

El $100 \%$ del personal administrativo expresaron que si mantienen buenas relaciones con el gerente. Un aspecto positivo, que de hecho debería facilitar la estrecha colaboración entre ambos grupos para el logro de la misión y los objetivos de la empresa. 
5.- ¿Considera usted que la cooperativa cumple con las expectativas de los usuarios?

\section{Cuadro 14.- CUMPLIMIENTO CON LAS EXPECTATIVAS DE LOS USUARIOS}

\begin{tabular}{|l|c|c|}
\hline OPCIONES & FRECUENCIA & $\%$ \\
\hline SI & 69 & $48 \%$ \\
\hline NO & 74 & $52 \%$ \\
\hline Total & 143 & 100 \\
\hline
\end{tabular}

Un 52\% del personal administrativo piensa que la cooperativa si cumple con las expectativas de los usuarios, y un $48 \%$ piensa que no. Hay una mínima diferencia entre ambos porcentajes, lo que indica que también entre las personas que integran el personal de las cooperativas de transporte urbano existe la duda de que el usuario este contento con el servicio que recibe.

6.- ¿Cree usted que las cooperativas de transporte urbano cumplen las normativas legales que los regulan?

Cuadro 15.- CUMPLIMIENTO CON LAS NORMATIVAS LEGALES QUE REGULAN A LAS COOPERATIVAS

\begin{tabular}{|l|c|c|}
\hline OPCIONES & FRECUENCIA & $\%$ \\
\hline SI & 126 & $88 \%$ \\
\hline NO & 17 & $12 \%$ \\
\hline Total & 143 & 100 \\
\hline
\end{tabular}

Un $88 \%$ del personal administrativo cree que las cooperativas de transporte urbano cumplen con las normativas legales que las regulan, y un $12 \%$ piensa que no. Al parecer el personal está muy seguro de que si cumplen con las normativas legales de tránsito.

Entrevista dirigida a los gerentes que dirigen las cooperativas de transporte urbano de la ciudad de Babahoyo 
Wendy L. Ocampo-Ulloa; Washington Pazmiño-Gavilanez; Veronica A. Merchan-Jacome

\section{P ¿Cuál es la misión de la cooperativa que usted dirige?}

Las cooperativas de transporte urbano de la ciudad de Babahoyo tienen como misión brindar un servicio de calidad a todos los usuarios.

\section{$\mathbf{R}$ ¿Planifican la actividad administrativa y de servicio de la cooperativa?}

Si se planifica, para eso está designado un consejo de Administración que se reúne frecuentemente con el fin de planificar estrategias para encaminar a nuestras cooperativas al éxito, y vale recalcar que no solo el consejo administrativo participa en la planificación de ideas sino también todos los socios que conforman esta cooperativa aportan con ideas valiosas para fortalecer esta institución.

Respecto a los recorridos y rutas ya están establecidos por la Agencia Nacional de Transporte y eso no se puede cambiar, lo que si se planifica es cuando existe un daño de carreteras u obstáculos que impidan la ruta normal.

\section{P ¿Qué opinión cree usted, poseen los usuarios del servicio que presta la cooperativa?}

R Sabemos que la ciudadanía siempre va a estar inconforme con el servicio que prestamos, ya que nuestros usuarios son muy exigentes y críticos, pero para nosotros eso es algo bueno ya que en base a sus críticas, nosotros podemos seguir trabajando y coordinando con el fin de ofrecer un servicio óptimo a la ciudadanía.

\section{$P_{\text {¿Capacita frecuentemente al personal de las unidades de transporte? }}$}

R Bueno la capacitación en nuestra cooperativa se la realiza frecuentemente ya que nuestro personal de trabajo necesita estar capacitado para realizar sus actividades, nos enfocamos en 
La gestión administrativa de las empresas de transporte urbano de Babahoyo y sus efectos en el grado de satisfacción de los usuarios

atención al cliente y relaciones humanas debido a que en nuestro medio de trabajo nuestros empleados necesitan brindar una buena atención y tener una muy buena relación con los usuarios, también les brindamos charlas de motivación ya que una persona debe estar motivada para realizar su trabajo con éxito.

\section{P ¿Considera usted que las unidades de transporte cumplen con los parámetros de la ley?}

R Si el $90 \%$ de nuestras unidades han sido remodeladas y todas cumplen con los parámetros y medidas establecidas por la Agencia Nacional de Tránsito. Y el 10\% restante ya se encuentran en proyecto de cambio.

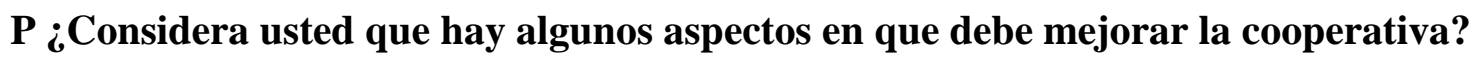

R Considero que si hay aspectos que hay que mejorar considerando que nuestros usuarios son muy exigentes, pero estamos trabajando para día a día mejorar el servicio que brindamos.

\section{P ¿Mantiene buenas relaciones con el personal administrativo y de servicio?}

R Si se mantiene una excelente relación con el personal administrativo y de servicio ya que pienso que una buena comunicación es la base fundamental para resolver los conflictos existentes en nuestra cooperativa.

\section{P ¿Qué política mantiene dentro de la cooperativa a fin de brindar un buen servicio al usuario?}

R Las políticas de nuestra cooperativa son muy exigentes en cuanto al aseo de nuestras unidades y del personal que labora en estas, también trabajamos mucho en el buen trato al usuario y 
Wendy L. Ocampo-Ulloa; Washington Pazmiño-Gavilanez; Veronica A. Merchan-Jacome

la forma de desempeñar su trabajo, nos encargamos de emitir multas y sanciones a nuestros trabajadores a fin de que no se vuelva a cometer el mismo error.

\section{Conclusiones.}

De las investigaciones realizadas se han llegado a las siguientes conclusiones:

El modelo de gestión administrativa de las empresas de transporte urbano actualmente utilizado, no es el adecuado para la satisfacción del usuario.

La calidad de servicio que brindan las cooperativas de transporte urbano es regular, esto explica porque los usuarios se sienten insatisfechos con el servicio que se les presta.

La condición de las unidades de transporte no ofrecen comodidad, seguridad a la integridad física y al entorno ambiental del usuario.

El trato que se proporciona en las unidades de transporte urbano no son las adecuadas para satisfacer al usuario.

El personal administrativo necesita ser más capacitado en temas actuales relacionados a la administración y calidad total.

Algunos miembros del personal administrativo desconoce cuál es la misión de la cooperativa por lo que no colaboran adecuadamente al cumplimiento de la misma.

Los principales factores que influyen en el grado de satisfacción del usuario son las malas condiciones en que se prestan el servicio y el ambiente desfavorable para la prestación del mismo. 
La gestión administrativa de las empresas de transporte urbano de Babahoyo y sus efectos en el grado de satisfacción de los usuarios

Una aproximación real al servicio demuestra que el mismo es insuficiente para cumplir con

las expectativas de los usuarios.

\section{Bibliografía.}

Egaña Ugrinovic, J., Ortúzar, J., \& Rizzi, L. (2009). Inclusión de variables latentes en modelos de elección discreta para usuarios de buses y trenes interurbanos. XIV Congreso Chileno de Ingeniería de Transporte, (págs. 1-14). Concepción.

Heredia Bedoya, J. (2015). Modelo de satisfacción de los usuarios de transporte público tipo bus integrando variables latentes. Facultad de Minas. Medellín: Universidad Nacional de Colombia.

Ortúzar, J., \& Román, C. (2003). El problema de modelación de demanda desde una perspectiva desagregada: el caso del transporte. EURE, 29(1), 149- 171.

Ortúzar, J., \& Willumsen, L. (2008). Modelos de transporte. Madrid: Vasa.

Yáñez, M., Raveau, S., \& Ortúzar, J. (2010). Inclusion of latent variables in Mixed Logit models: Modelling and forecasting. Transportation Research Part A: Policy and Practice, 44(1), 744-753. 